\title{
Stability of Cubic $\mathrm{FAPbl}_{3}$ from X-Ray Diffraction, Anelastic and Dielectric Measurements
}

\author{
Francesco Cordero, ${ }^{*, \dagger}$ Floriana Craciun, ${ }^{\dagger}$ Francesco Trequattrini, ${ }^{\ddagger}, \dagger$ Amanda \\ Generosi, ${ }^{\dagger}$ Barbara Paci, ${ }^{\dagger}$ Anna Maria Paoletti, ${ }^{\circledR}$ and Giovanna Pennesi ${ }^{\Uparrow}$ \\ $\dagger$ †stituto di Struttura della Materia-CNR (ISM-CNR), Area della Ricerca di Roma - Tor \\ Vergata, Via del Fosso del Cavaliere 100, I-00133 Roma, Italy \\ $\ddagger$ Dipartimento di Fisica, Università di Roma "La Sapienza", p.le A. Moro 2, I-00185 \\ Roma, Italy \\ -Istituto di Struttura della Materia-CNR (ISM-CNR), Area della Ricerca di Roma 1, Via \\ Salaria, Km 29.300, I-00015 Monterotondo Scalo, Roma, Italy \\ E-mail: francesco.cordero@ism.cnr.it
}

\begin{abstract}
Among the hybrid metal-organic perovskites for photovoltaic applications $\mathrm{FAPbI}_{3}$ (FAPI) has the best performance regarding efficiency and the worst regarding stability, even though the reports on its stability are highly contradictory. In particular, since at room temperature the cubic $\alpha$ phase, black and with high photovoltaic efficiency, is metastable against the yellow hexagonal $\delta$ phase, it is believed that $\alpha$-FAPI spontaneously transform into $\delta$-FAPI within a relatively short time. We performed X-ray diffraction and thermogravimetric measurements on loose powder of FAPI, and present the first complete dielectric and anelastic spectra of compacted FAPI samples under various conditions. We found that $\alpha$-FAPI is perfectly stable for at least 100 days, the duration of the experiments, unless extrinsic factors induce its degradation. In our tests, degradation was detected after exposure to humidity, strongly accelerated by grain boundaries and the presence of $\delta$ phase, but it was not noticeable on the loose powder kept in air under normal laboratory illumination. These findings have strong implications on the strategies for improving the
\end{abstract}

stability of FAPI without diminishing its photovoltaic efficiency through modifications of its composition.

\section{Graphical TOC Entry}

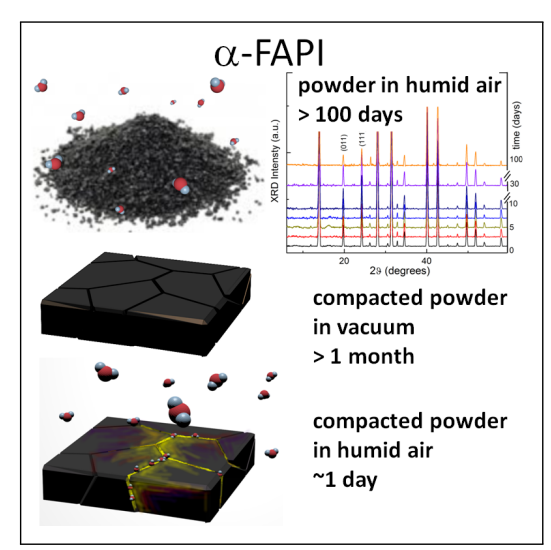


Although $\mathrm{MAPbI}_{3}$ (MAPI, MA = methylammonium $\mathrm{CH}_{3} \mathrm{NH}_{3}$ ) is the most studied hybrid metal-organic perovskite for photovoltaic applications, ${ }^{1}$ better performance in terms of photovoltaic efficiency are found in $\mathrm{FAPbI}_{3}$ (FAPI, $\mathrm{FA}=$ formamidinium $\left.\mathrm{CH}\left(\mathrm{NH}_{2}\right)_{2}\right)$. This is due both to a smaller bandgap of FAPI and to the fact that the $\mathrm{FA}^{+}$ion, in spite of a smaller electric dipole with respect to $\mathrm{MA}^{+}$, has a much larger quadrupole and faster reorientation dynamics that better screen the photoexcited carriers, enhancing their lifetime. ${ }^{2}$ FAPI also has a better stability than MAPI at high temperature but its major flaw is that the black cubic $\alpha$ phase, which has the high photovoltaic efficiency, is metastable at room temperature, where instead the stable phase, and the one obtained by standard chemical methods, is the yellow hexagonal $\delta$ phase. For these reasons, major efforts are directed now at trying to stabilize the cubic $\alpha$ phase of FAPI through partial substitutions of FA with MA, Cs, etc. or I with $\mathrm{Br}$, although this approach increases the bandgap. $\frac{314}{1 t}$ has been discussed, based on neutron diffraction measurements and simulations, that the $\alpha \rightarrow \delta$ transformation is complex and occurs through various intermediate stages, requiring to overcome a free energy barrier estimated in the order of hundreds of milli-electron volt. ${ }^{5}$ This explains why the $\alpha$ phase of FAPI is kinetically trapped, resulting in a large thermal hysteresis between the $\delta \rightarrow \alpha$ transition at $T_{\delta \alpha}^{h}=350 \mathrm{~K}$ and the $\alpha \rightarrow \delta$ at $T_{\delta \alpha}^{c}=290 \mathrm{~K} . \underline{5}$ Actually, the barrier for the $\alpha \rightarrow \delta$ transition has not been measured, and there is complete uncertainty on the kinetics of this transition. Indeed, also the reported temperatures for the $\delta \rightarrow \alpha$ transition during heating span quite a broad range between $350 \mathrm{~K}^{5}$ and $458 \mathrm{~K}, \frac{6}{6}$ and this may be partly explained with the slow kinetics of the transformation, since the lowest temperature is observed in a quasistatic neutron scattering experiment while the highest during fast heating at $5 \mathrm{~K} / \mathrm{min}$. The uncertainties on the reverse transformation are worse. The reported stability of the $\alpha$ phase at room temperature in humid or dessicated air ranges from few hours, $7-11$ to few days, $12-14$ but stability over months has been achieved by grow- ing FAPI in a solution with long chain alkyl or aromatic ammonium cations which cover the (100) grain surfaces and render them stable, 4 or by growing FAPI within template alumina nanotubes. 14 At variance with studies that highlight the role of defects and grain boundaries in stimulating degradation, $15[16$ it is also believed that nanostructured FAPI has a superior stability. ${ }^{17 / 18}$ Closely connected with this issue is the variety of results obtained in different experiments using a technique as effective as neutron diffraction for studying the nature of the polymorphism of FAPI, as recently summarized in Ref. 19.

We will show that a loose micrometric powder of FAPI, prepared by slightly modifying the known procedures can remain exposed in air at normal laboratory illumination for at least 100 days without any sign of degradation and pressed pellets can likewise remain in the $\alpha$ phase for at least one month after being thoroughly dried in high vacuum. This observations suggest that the kinetic trapping of the $\alpha$ phase of FAPI at room temperature lasts for much longer than previously thought, and the transformation to the $\delta$ phase, itself highly hygroscopic, is catalyzed by humidity together with other extrinsic factors, such as grain boundaries.

FAPI samples were obtained following an already reported procedure ${ }^{20}$ with slight modifications, by precipitating with toluene the yellow phase from a solution of $\mathrm{HC}\left(\mathrm{NH}_{2}\right) \mathrm{I}_{2}$ and $\mathrm{PbI}_{2}$ in GBL. The yellow powder was recovered by centrifugation and put under vacuum at $150 \div 160{ }^{\circ} \mathrm{C}$ for $\sim 4 \mathrm{~h}$ until completely transformed into dark grey/black $\alpha$-FAPI (details in SI).

We performed four types of experiments on two types of samples: X-ray diffraction (XRD) in air at room temperature on loose powder and also on the compacted samples; complex Young's modulus versus temperature in high vacuum on a compacted thin bar (FAPI-b) and dielectric permittivity versus temperature in a small closed volume on compacted discs (FAPI$\mathrm{d} 1$ and FAPI-d2). The results on the stability of $\alpha$-FAPI turned out to be strongly dependent on the different experimental conditions. 
In order to test whether traces of water or other solvents were present in the freshly prepared powders, we performed Thermo Gravimetric Analysis (TGA) and Differential Scanning Calorimetry (DSC) on 20 - 30 mg batches of $\delta$-FAPI-before and after its conversion to $\alpha$-FAPI. The results are shown in Fig. S1 and no mass loss attributable to water or other solvents could be detected until the sample decomposition started above $250{ }^{\circ} \mathrm{C}$. The yellow samples exhibited a dip in the DSC signal at $\gtrsim 150{ }^{\circ} \mathrm{C}$, due to the $\delta \rightarrow \alpha$ conversion.

An unexpected positive result was the perfect stability for at least 97 days of the loose powder of $\alpha$-FAPI in air under normal laboratory illumination with neon lamps. In Fig. 1 the XRD spectra taken over a period of 100 days did not change at all (during the 2 months before the last measurement the powder was kept in a closed polypropylene vial). The initial pattern was acquired few hours after the conversion of the $\delta$-FAPI powder into $\alpha$-FAPI. The particles had sizes exceeding the micrometer, as deduced from the narrow reflections, whose width was determined by the XRD setup (SI). This is plain evidence that the kinetics of the $\delta \rightarrow \alpha$ transition in bulk $\alpha$-FAPI at room temperature is much slower than generally assumed, even without the often cited stabilization size effect in nanometric particles. $\frac{17 \mid 18}{}$ This is true not only above $T_{\delta \alpha}^{c} \simeq 290 \mathrm{~K}$ deduced from neutron diffraction, ${ }^{5}$ but also including one week at several degrees below that temperature. The stability against decomposition is likewise high, since no trace of $\mathrm{PbI}_{2}$ is detected. The first sign of degradation through reversion to the $\delta$ phase has been found after additional 45 days (Fig. S2).

On the other hand, the small part of powder that remained attached to the walls of the glass bottle rapidly became yellow (SI).

The pressures at which we compacted the discs and the bars were 0.62 and $0.2 \mathrm{GPa}$ respectively, in the range of structural transitions of $\alpha$-FAPI reported in the literature, notably to the $\delta$ phase, $\stackrel{23}{ }$ even though there is disagreement. $\frac{2425}{21}$ Indeed, the presence of $\delta$-FAPI in the pressed samples could be determined both visually, as yellow spots (see Fig. S3), and from

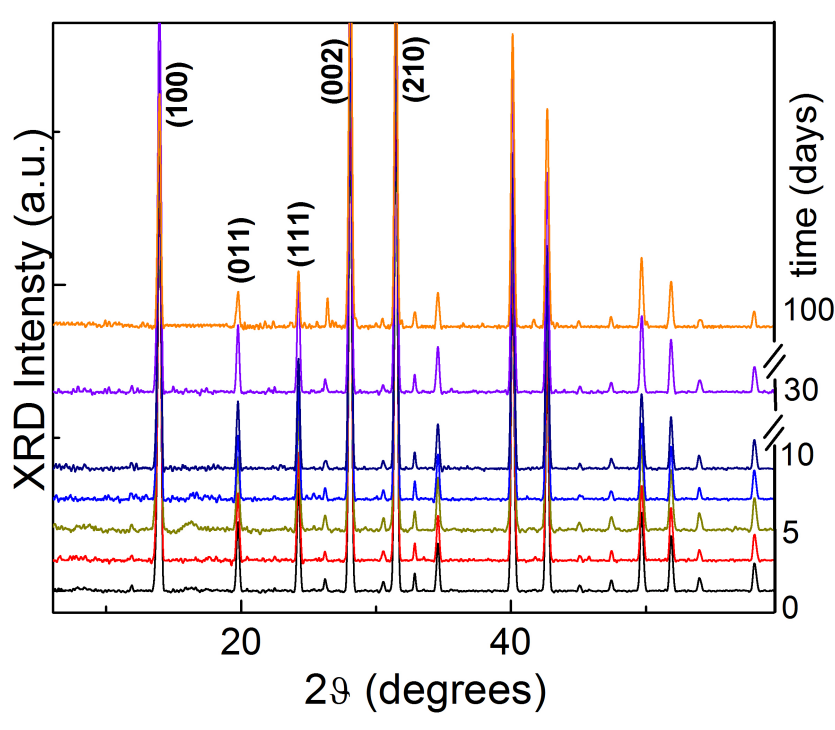

Figure 1: XRD patterns collected from the precursor FAPI powder at different times. The $\alpha$-FAPI cubic phase was detected and labeled according to the literature. 21122

the XRD analysis of the disc FAPI-d2 (Fig. S2). It seems that also grinding the powder of originally pure $\alpha$-FAPI in air rapidly induced a transformation to $\delta$-FAPI. In fact, being the first attempts to make a bar unsuccessful, we repeatedly pressed and reground the powder in an agate mortar, observing a progressive enrichment in yellow grains. The bar finally used in the anelastic experiments (FAPI-b) was pressed from freshly prepared powder of $\alpha$-FAPI in a single successful attempt.

The anelastic measurements were performed in a vacuum of $<10^{-5}$ mbar at high temperature or in $<0.2$ mbar He at low temperature, and the system was left in static vacuum between the experiments. Details on the technique and sample conditions are found in SI. The anelastic spectrum of FAPI has been measured here for the first time, similarly to the complete dielectric spectrum, and both are used here for the specific task of evaluating the fraction of $\alpha$ phase in our samples. The conclusions that can be drawn from these spectra on the nature of the structural transitions that $\alpha-\mathrm{FAPI}$ undergoes are left for future work.

Figure 2 presents most of these measurements as Young's modulus $E$ normalized to the value $E_{0}$ initially reached in the $\alpha$ phase. During the first heating, a sudden rise of the modulus at 


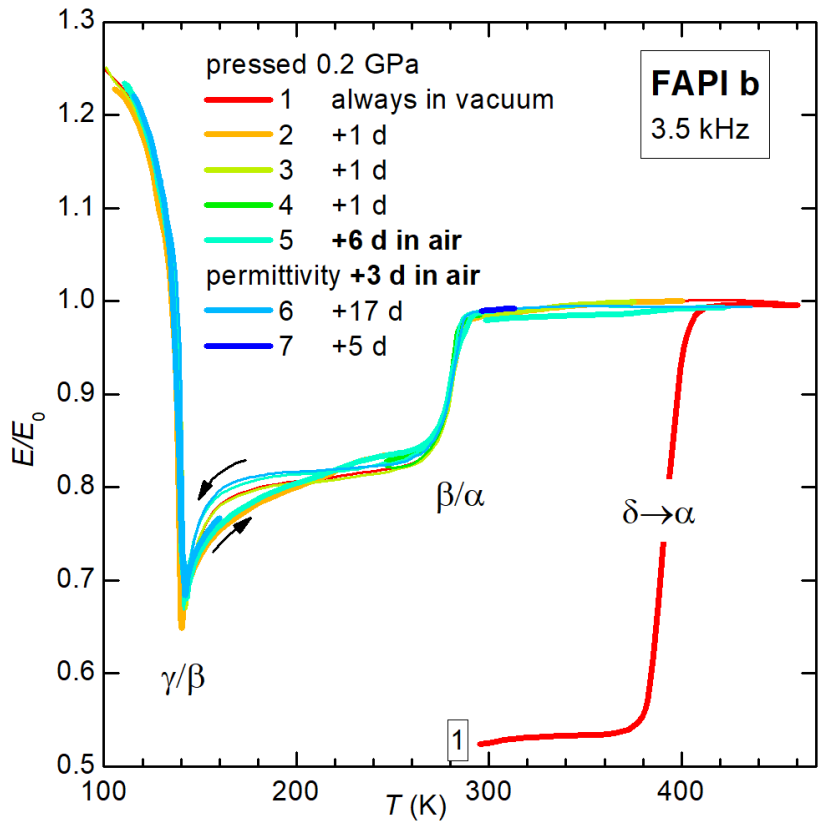

Figure 2: Young's modulus, normalized to the initial value in the $\alpha$ phase, measured at $3.5 \mathrm{kHz}$ over several temperature cycles during 35 days. The sample was always in vacuum, except for 6 days between curves 4 and 5 and additional 3 days for measuring the dielectric permittivity before curve 6 . See SI for details.

$\sim 390 \mathrm{~K}$ must correspond to the transformation of the $\delta$ phase induced by pressure back to the $\alpha$ phase, after which the sample remains in the $\alpha$ phase for all the subsequent temperature cycles and agings at room temperature. When cooling, the $\alpha$ phase transforms into $\beta$ at $280 \mathrm{~K}^{26}$ and then into $\gamma$ at $141 \mathrm{~K} .26$ The anomalies occurring at these temperatures are retraced during heating, except for some thermal hysteresis above the $\gamma \rightarrow \beta$ transition, as indicated by the arrows. It is not the aim of this paper to analyze the elastic anomalies at these phase transitions; we will only say that the steplike softening at the $\alpha \rightarrow \beta$ transition is as expected for a transition where the $\mathrm{PbI}_{6}$ octahedra tilt with respect to the symmetric cubic phase (strain is coupled to the square of the tilt angle $\mathrm{e}^{27}$ ), and is analo-

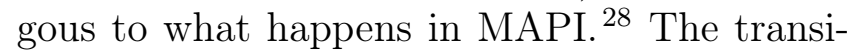
tion to the $\gamma$ phase is more enigmatic, defined as reentrant psudocubic ${ }^{[26}$ with near cancelation of the octahedral tilting and presumably freezing of the reorientations of the FA molecules. Such a freezing would explain the restiffening of the elastic modulus well above the value of the cubic phase, and is similar to what happens below the analogous transition in MAPI, although in that case the reorientational freezing is mainly thermally activated and occurs below the transition to the $\gamma$ phase.

The interesting point in the present context is that, after the initial $\delta \rightarrow \alpha$ transformation, the $E(T)$ curves remain perfectly stable for 1 month, and this excludes that any transformation to the $\delta$ phase or degradation occurs over the month time scale in vacuum. A minor degradation, indicated by $\lesssim 1 \%$, decrease of $E$, occurred during a total of 9 days in air between curves 4 and 6 , during which the dielectric permittivity was also measured. Details are found in the SI.

Few days after completing the cycle of anelastic measurements, the XRD spectra showed that a rapid degradation processes had started. The $\alpha$ phase was still present but the dominant peak was (001) of $\mathrm{PbI}_{2}$ (Fig. S2). The high degree and irreversibility of degradation was confirmed by a new cycle of anelastic measurements (Fig. S6) with an extremely low Young's modulus that could not be recovered by annealing above $400 \mathrm{~K}$.

The complex dielectric permittivity was measured in a Linkam stage with modified Examina Probe having a sealed volume of $\sim 100 \mathrm{~cm}^{3}$. The standard procedure for removing humidity is flushing the probe with a dry gas evaporated from liquid nitrogen or air, for few minutes at $40{ }^{\circ} \mathrm{C}$, then the probe volume is sealed and the measurement starts. The permittivity $\epsilon(T)$ curves measured two hours after pressing the powder into disc are shown in Fig. 3 for the sample FAPI-d2. The permittivity had an initially low value $\lesssim 20$, which sharply raised above 45 around $400 \mathrm{~K}$, the temperature of the $\delta \rightarrow \alpha$ transformation. Heating was stopped at $430 \mathrm{~K}$ in order to avoid the thermal degradation of the organic component and immediately followed by cooling at $-1.2 \mathrm{~K} / \mathrm{min}$. During cooling the permittivity remained high and presented two anomalies at the structural $\alpha \rightarrow \beta$ and $\beta \rightarrow \gamma$ transitions, in agreement with the anelastic measurements. There were also an additional anomaly at $173 \mathrm{~K}$ and some struc- 
ture below the $\beta / \gamma$ transition, which were reproducible over several temperature cycles on two different samples.

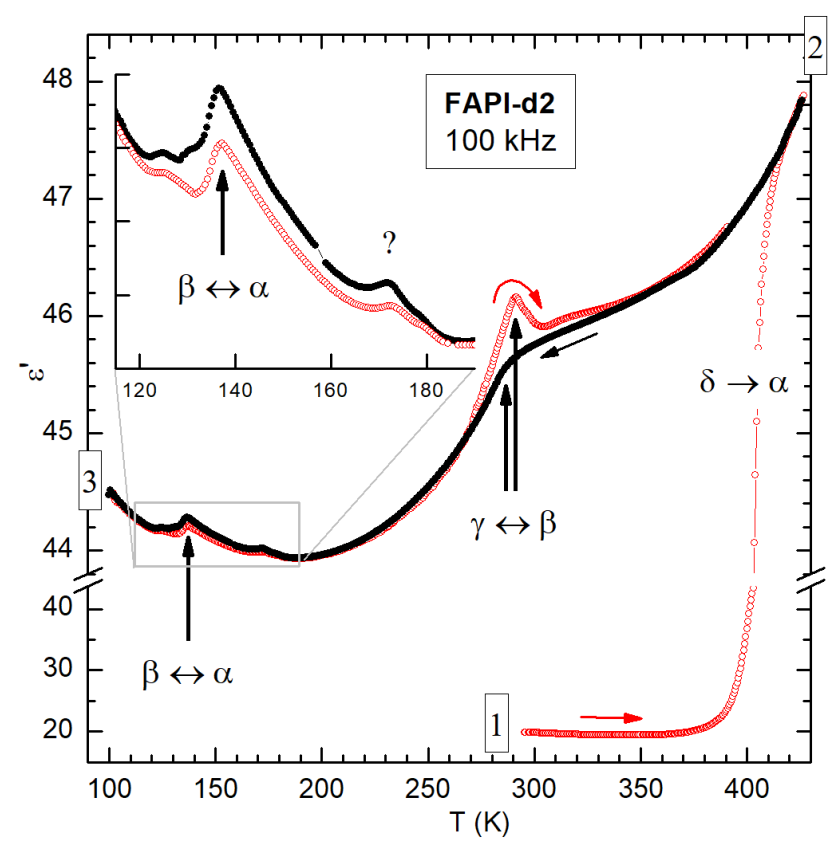

Figure 3: Real part of the permittivity of FAPI measured at $100 \mathrm{kHz}$ during (1) heating at $1.2 \mathrm{~K} / \mathrm{min} 2 \mathrm{~h}$ after pressing the disc, (2) cooling at $-1.2 \mathrm{~K} / \mathrm{min}$ and (3) heating at $1.5 \mathrm{~K} / \mathrm{min}$. Note the broken vertical scale.

The $\epsilon(T)$ curve during heating perfectly reproduces the cooling, except for the peak/step at $T_{\beta \alpha}$, which is sharper and higher, but this is probably an effect of incomplete removal of humidity, as discussed below. Indeed, if some humidity is present during cooling, it condenses and freezes on the sample and increases the conductivity and permittivity after becoming liquid during subsequent heating.

If humidity was removed with the standard procedure described above, after one day the permittivity dropped again to values even lower than the initial one of mixed $\alpha$ and $\delta$ phase. This is shown in Fig. 4 reporting results on FAPI-d1. The experiment was repeated twice at one day interval (curves 2 and 3), yielding the same result, and after four days (4) the starting permittivity was even lower, until the sample was removed. Its border free from Ag was still black and was analyzed by XRD (Fig. S3), which indicated a strongly degraded $\alpha$ phase, without any sign of $\delta$ phase.

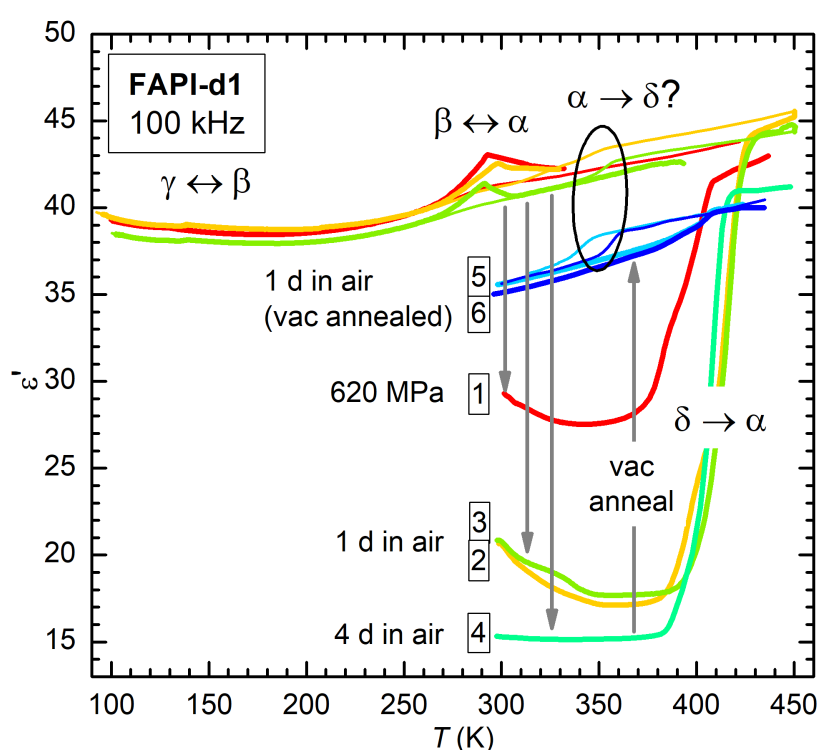

Figure 4: Real part of the permittivity of FAPI measured at $100 \mathrm{kHz}$ during several temperature cycles using the standard procedure for removing humidity at $40{ }^{\circ} \mathrm{C}$. After vacuum annealing, $\epsilon$ is more stable at its higher level.

Then the sample was annealed for $5 \mathrm{~min}$ in a vacuum of $10^{-4}$ mbar at $\lesssim 200{ }^{\circ} \mathrm{C}$ and immediately mounted in the dielectric probe with the standard procedure for flushing out the humidity. This time the initial permittivity was high (5), and remained so when repeating the experiment the following day (6). During all these temperature runs there was a progressive overall decrease of the $\epsilon(T)$ curves in the $\alpha, \beta$ and $\gamma$ sequence of phases, indicating that some irreversible damage to the sample occurred after each cycle. There is also a small step when cooling through $350 \mathrm{~K}$, whose amplitude increases at every cycle; it is tentatively indicated as partial $\alpha \rightarrow \delta$ transition. These results strongly suggest that humidity is stored in the sample, cannot be removed by the standard purging procedure at $40{ }^{\circ} \mathrm{C}$ and is responsible for the degradation of the $\alpha$ phase, though initially without full conversion to the $\delta$ phase. Above the $\delta \rightarrow \alpha$ transition temperature, most of the water is expelled by the sample, but remains trapped into the closed volume of the probe, so promoting again the degradation of the $\alpha$ phase already during the hours following the measurement. Water was instead removed by the short treatment at high temperature in vacuum be- 
fore measuring the last curves 5 and 6 .

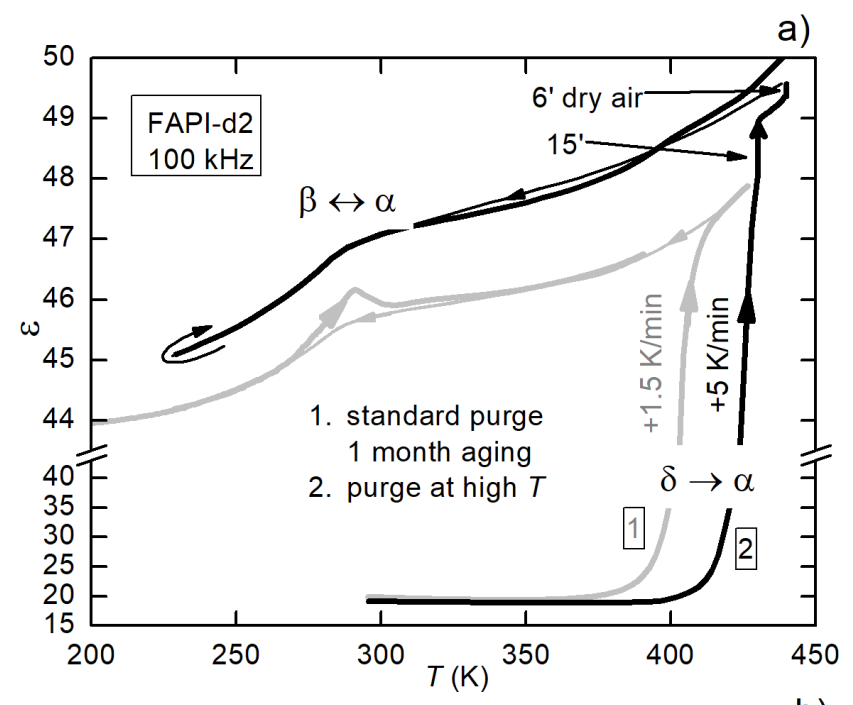

b)

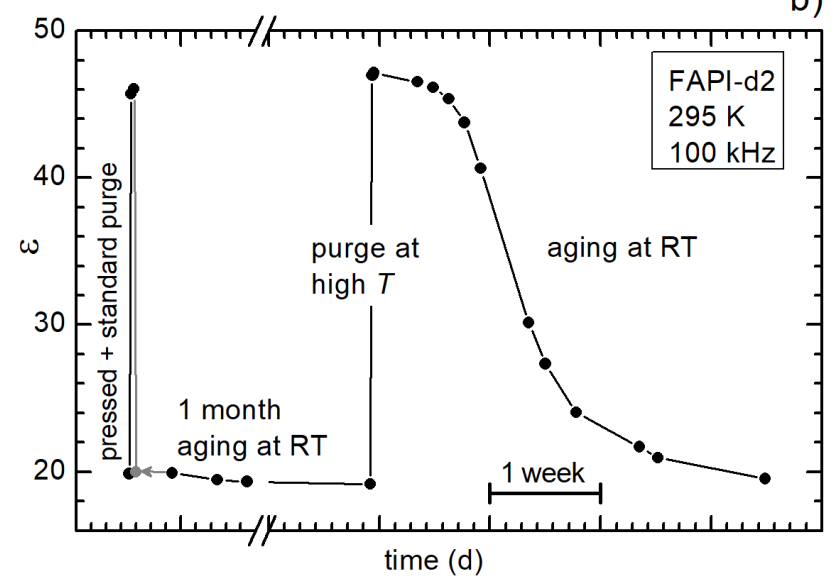

Figure 5: Real part of the permittivity of FAPI$\mathrm{d} 2$ measured at $100 \mathrm{kHz}$ during two temperature cycles and aging. (a) $\epsilon(T)$ curves measured (1) just after pressing the sample with standard purging from humidity ar $313 \mathrm{~K}$ (same as Fig. 3) and (2) after aging 1 month and purging at $440 \mathrm{~K}$. Notice the broken vertical scale. $\epsilon$ measured at $295 \mathrm{~K}$ and $100 \mathrm{kHz}$ versus time.

In order to confirm this hypothesis, we repeated the measurement of sample FAPI-d2 with an improved drying procedure. Figure 5 a shows the previous measurement of Fig. 3 (gray curves 1) with the standard drying procedure followed by one month aging and the new measurement (black curves 2) with a protocol where purging is carried out during $6 \mathrm{~min}$ at $440 \mathrm{~K}$, above the $\delta \rightarrow \alpha$ transition (details in the SI). As expected, the latter procedure was much more effective in removing water from both the sample and the probe volume: it yielded a more complete transformation into the $\alpha$ phase, as demonstrated by the higher level reached by the permittivity, and greatly extended the stability in time of the $\alpha$ phase, as shown in the lower panel. As explained in the SI, the degradation kinetics was more than 30 times slower than previously, and longer dehydration treatment would have certainly further improved the stability of the $\alpha$ phase.

The experimental results described above can be summarized as follows:

1) According to XRD the loose powder remains stable in the $\alpha$ phase in normally humid air at room temperature for at least 100 days.

2) Pressing the powder of pure $\alpha-$ FAPI into bars or discs at $0.2-0.6 \mathrm{GPa}$ for few minutes induces a partial transformation to the $\delta$ phase and rapid absorption of humidity. The partially degraded phase reverts to $\alpha$ during the first heating above $400 \mathrm{~K}$, but the subsequent results on the pressed samples depend on whether the measurements are carried out in dynamic high vacuum or in a small closed volume.

3) According to the anelastic experiments in high vacuum, the compacted powder remains stable in the $\alpha$ phase for at least one month, including one week in air, but after further exposure to air it rapidly degrades.

4) According to the dielectric experiments in a small closed volume purged from humidity before each experiment at $313 \mathrm{~K}$, most of the compacted powder remains stable in the $\alpha$ phase during the full temperature run from $450 \mathrm{~K}$ down to $100 \mathrm{~K}$ and heating again to above room temperature. However, already after half day the dielectric permittivity drops back to the original state of partially degraded $\alpha$ phase, with a seeming saturation of the degradation process after few days. Yet, repeating the full temperature cycles reproduces the first experiment, and the whole process of transformation into $\alpha$ phase and partial degradation can be repeated several times. The stability of the $\alpha$ phase is enhanced after purging from humidity is made more effectively at higher temperature.

$5)$ the yellow $\delta$ phase is an advanced stage in the paths to degradation in the presence of humidity and the dielectric constant and elastic modulus are strongly sensitive also to the ini- 
tial and partly reversible stages of degradation, without visible formation of yellow phases.

At first these observations may seem to add puzzling information to the already rich known phenomenology, but they can all be easily explained and harmonized with the existing literature.

The first strong conclusion that can be drawn from 1) and 3 ) is that $\alpha$-FAPI, although metastable at room temperature, is much more stable than previously thought, over a time scale of months and possibly more, depending on the extrinsic factors that induce the $\alpha \rightarrow \delta$ transformation and/or degradation into $\mathrm{PbI}_{2}$ with loss of the organic part. The nature of these extrinsic factors can be deduced form the above observations and finds confirmation in the literature.

The discs and bars were pressed at 0.6 and $0.2 \mathrm{GPa}$, respectively, and the observation of diffraction peaks of the $\delta$ phase and of yellow grains is in accordance with the recent report that pressure induces the $\alpha \rightarrow \delta$ transition at room temperature already at $<0.1 \mathrm{GPa} .23$ On the other hand, there is no agreement with the few other cases where the effect of pressure on the structure of $\alpha$-FAPI has been studied: no transition has been found up to $2.5 \mathrm{GPa}, 25$ or up to $0.6 \mathrm{GPa}$ in colloidal FAPI nanocrystals ${ }^{18}$ or transitions to different phases have been observed, $\stackrel{18|24| 29}{ }$ while nanocrystalline inorganic halide perovskites exhibit better stability. $\underline{30[31}$ Among all these data, we find agreement with those or Ref. 23 and, regarding the variety of the available results, we observe that in the small volumes of the high pressure experiments the issues discussed below related to adsorbed water or solvents are greatly amplified.

Humidity is perhaps the most acknowledged cause of degradation of the metal-organic perovskites, ${ }^{32}$ but there is extreme variability in the rate and nature of degradation of FAPI, as mentioned in the introduction and also within the results presented here. The effect of humidity has been much studied in MAPI, where two different types of degradation have been recognized, depending whether water condenses at the perovskite surface or not. Condensed water irreversibly decomposes MAPI into $\mathrm{PbI}_{2}$ and $\mathrm{HI}$ with the organic molecule dissolved in water or lost as gas. In addition, the reaction sequence seems autocatalytic through the degradation products,,$\sqrt[33]{3}$ so that beyond a certain point it becomes very fast. On the other hand, if water does not condense it diffuses in the bulk and forms hydrated compounds where the network of alkali octahedra is partially broken and intercalated with the organic and $\mathrm{H}_{2} \mathrm{O}$ molecules; 34 this type of degradation during a first stage can be reversed by drying the material. ${ }^{32}$

In FAPI, as in other materials, water condensation should occur preferentially at grain boundaries. For example in gold, it has been observed that grain boundaries and terraces on the surface are preferential sites for water absorption, $\frac{35}{35}$ presumably due to their favorable topographic conformation. In addition, grain boundaries often have a higher density of defects, which in the case of the metal-organic perovskites might favor both the adsorption and reaction of water molecules. Indeed in FAPI films there are both indirect and direct evidence in this sense. Voids attributed to the condensation of water during preparation are found to run along the grain boundaries,, 36 and the degradation in FAPI films exposed to humidity has been observed via Kelvin probe force microscopy to initiate at grain boundaries. ${ }^{16}$ In addition, combined scanning TEM and energy loss spectroscopy detected the presence of $\mathrm{OH}^{-}$ ions at the FAPI grain boundaries, a sign of reacted water. 15

With these premises, the above observations 1) - 4) become clear. The superior stability, even in humid air, of $\alpha$-FAPI in loose powder with respect to the compacted form is explained with the fact that the powder has few grain boundaries, if any, whereas in the compacted powder, as in films, each grain is circumscribed by grain boundaries acting as water condensation centers.

Both types of degradation under humidity described above, reversible intercalation of $\mathrm{H}_{2} \mathrm{O}$ and subsequent decomposition, considerably lower the elastic moduli and dielectric constant, because they break the network of $\mathrm{Pb}-\mathrm{I}$ bonds and eliminate the polyhedral spaces between 
octahedra, where the organic molecules are free to reorient. It is therefore impossible to distinguish among the various stages of hydration and occurrence of partial $\alpha \rightarrow \delta$ transformation from sets of curves like those of Figs. $1-3$. The positive step in $\epsilon$ and $M$ when heating through $400 \mathrm{~K}$ is labeled $\alpha \rightarrow \delta$ because it corresponds to the known temperature of that transition, but probably it includes the contribution from dehydration of the material. Analogously, the smaller step sometimes observed during cooling in $\epsilon(T)$ is labeled " $\alpha \rightarrow \delta$ ? ", but it must also include rehydration from the water remained trapped in the closed volume of the dielectric probe. The hydration and subsequent reactions are frozen during cooling to lower temperatures, but proceed when back to room temperature, where $\epsilon$ drops to less than half within the next day. The proof that water drives the degradation process is given by the prolonged stability of the $\alpha$ phase during aging at room temperature (curve 2 in Fig. 5 a and Fig. 5b) after the improved dehydration of the sample and elimination of water from the closed volume. A similar result is obtained by a preventive drastic dehydration at $10^{-4} \mathrm{mbar}$ and $200^{\circ} \mathrm{C}$ (curves 5,6 of Fig. 4). Moreover, the bar for the anelastic measurements remains stable in air for 9 days, after a thorough dehydration in high vacuum for few days. This is in accordance with results in films, where a thermal treatment after preparation is found to improve the stability. 16

Considering that the TGA runs do not show measurable losses of water on $\alpha$-FAPI powder, we must conclude that water is mainly adsorbed after pressing the powder, due to the formation of the grain boundaries and probably even more to a higher hygroscopicity of the $\delta$ phase, and is released only at high enough temperature, probably $\geq 400 \mathrm{~K}$. Therefore, if the outgassing of the sample is performed near room temperature or not at all, the adsorbed water remains in the sample and starts to be released only near or above the $\delta \rightarrow \alpha$ transition and, if trapped in a closed volume, it is readsorbed during cooling and promotes degradation and possibly the reverse $\alpha \rightarrow \delta$ transformation. This is what happened in our first dielectric runs (curves 1-4 of Fig. 4).
The conclusion drawn from this discussion is that, although $\alpha$-FAPI is metastable at room temperature, having $\delta$-FAPI a lower energy, the energy barrier for converting the $\alpha$ into the $\delta$ phase is so high that no measurable conversion occurs at room temperature for at least 100 days (results from XRD on powder). The $\alpha \rightarrow$ $\delta$ reaction is probably catalyzed by water, together with other degradation pathways, which include hydration and decomposition; the latter has been found to occur even spontaneously in vacuum at the surface of MAPI. 37

As pointed out in the Introduction, there is no consistency among the various reports on the stability of $\alpha$-FAPI in the literature. This may be due to several factors, such as different preparation protcols, different types of samples, testing conditions and methods. Yet, it seems that the major discrepancy between the present observations and the literature is with the careful investigation of FAPI powder by neutron diffraction in Ref. 5. In particular, in that experiment it is found that a large quantity $(10-12 \mathrm{~g})$ of FAPI powder undergoes both the $\delta \rightarrow \alpha$ transition at $350 \mathrm{~K}$ during heating and the $\alpha \rightarrow \delta$ at $290 \mathrm{~K}$ during cooling in a closed volume filled with He, whereas we do not observe the latter neither in vacuum nor in air, when starting from pure $\alpha$-FAPI. Instead, similarly to other neutron and X-ray diffraction experiments, ${ }^{19 / 26}$ the octahedral tilt transitions of the perovskite structure are observed during cooling. Considering the well controlled conditions and effectiveness of the experimental technique of Chen et al., ${ }^{5}$ the discrepancy calls for an explanation, which we think is in the similarity between the conditions of that experiment and of our dielectric measurements with incomplete purging of humidity. In fact, in both cases the starting phase is completely or partially $\delta$, most likely hydrated, so that the water expelled by the sample at high temperature remains trapped in the experimental volume and drives the partial reversion to the $\delta$ phase during cooling.

The picture presented here is also in agreement with the previous observation ${ }^{26}$ that the powder of $\alpha$-FAPI obtained after heating $\delta$ FAPI overnight in a vacuum oven at $120^{\circ} \mathrm{C}$ was 
stable for $20-30$ days, whereas heating $\delta$-FAPI in an evacuated ampoule under static vacuum yields a black powder that reverts to yellow after few minutes. In the latter case the considerable amount of water contained in $\delta$-FAPI remains completely trapped in the ampoule and drives the reverse $\alpha \rightarrow \delta$ reaction immediately after cooling to room temperature.

It would also be interesting to reconsider the experiments where the stability is found to be highly enhanced in nanocrystalline FAPI. The observations include stable photoluminescence of colloidal nanocrystals with protected surface, 17 where the stabilization of the cubic phase is explained as a general effect of the small size of the particles, which enhances the surface-to-volume ratio. $\frac{17 \mid 18}{}$ Similarly, the observations of stability over several months of FAPI nanostructures protected by long chain alkyl or aromatic ammonium cations has been explained in terms of change of balance between bulk and surface energy after the surface functionalization, $\stackrel{4}{ }$ while the stability of $\alpha$-FAPI embedded in alumina nanotubes has been explained with the fact that the latter limit the possibility of expansion of $\alpha$-FAPI during degradation. ${ }^{14}$ In view of the stability of our micrometric loose powder and thoroughly dried compacted powder, we suggest that also the stability of nanostructured FAPI might alternatively be explained in terms of protection of $\alpha$-FAPI from humidity by the surface treatments or by the nanotubes and in terms of reduced fraction of grain boundaries in these single nanocrystals.

Our observations show that, even if the $\alpha$ phase of FAPI is metastable with respect to the yellow $\delta$ phase, its kinetic trapping at room temperature lasts for much longer than previously thought. The present data set the period of perfect stability of $\alpha$-FAPI to at least 100 days, corresponding to the duration of our tests, but this time range exceeds seven months if previous experiments on FAPI embedded in alumina nanotubes ${ }^{14}$ or with functionalized surfaces $^{4}$ are reinterpreted in terms of protection against humidity. This stability also includes the absence of spontaneous decomposition.

It appears that by far the most important factor inducing degradation of $\alpha$-FAPI is humidity at grain boundaries, and that the procedures used for dehydrating the material are often insufficient. Since grain boundaries are also present in films, these findings should help in devising strategies for improving the stability of $\alpha$-FAPI without modifying its composition, particularly by improving the initial dehydration process and subsequent surface protection. This is especially true in the cases of intentional exposure to moisture during preparation in order to improve the grain morphology through solvent annealing. $38 / 39$

Acknowledgement The authors thank Paolo Massimiliano Latino (ISM-Tor Vergata) for his technical assistance, Sara Notarantonio (ISM-Montelibretti) for the assistance in the synthesis, Marco Guaragno (ISM-Tor Vergata) for his technical support in X-ray experiments, Dr. Cristina Riccucci for the DTA-DSC measurements and Dr. Gloria Zanotti for fruitful discussions.

\section{Supporting Information Avail- able}

The following files are available free of charge.

The following files are available free of charge.

- SuppInfo.pdf: Synthesis, Thermal Analysis of $\alpha-$ and $\delta$-FAPI; Pressing; X-ray diffraction, particle size, degraded samples; Complex Young's modulus method and data of degraded sample; Dielectric measurements.

\section{References}

(1) Kakavelakis, G.; Paradisanos, I.; Paci, B.; Generosi, A.; Papachatzakis, M.; Maksudov, T.; Najafi, L.; Castillo, A. E. D. R.; Kioseoglou, G.; Stratakis, E. et al. Extending the Continuous Operating Lifetime of Perovskite Solar Cells with a Molybdenum Disulfide Hole Extraction Interlayer. Adv. Energy Mater. 2018, 8, 1702287. 
(2) Chen, T.; Chen, W. L.; Foley, B. J.; Lee, J.; Ruff, J. C.; Ko, J. Y. P.; Brown, C. M.; Harriger, L. W.; Zhang, D.; Park, C. et al. Origin of Long Lifetime of Band-Edge Charge Carriers in OrganicInorganic Lead Iodide Perovskites. PNAS 2017, 114, 7519.

(3) Binek, A.; Hanusch, F. C.; Docampo, P.; Bein, T. Stabilization of the Trigonal High-Temperature Phase of Formamidinium Lead Iodide. J. Phys. Chem. Lett. 2015, 6, 1249 .

(4) Fu, Y.; Wu, T.; Wang, J.; Zhai, J.; Shearer, M. J.; Zhao, Y.; Hamers, R. J.; Kan, E.; Deng, K.; Zhu, X. Y. et al. Stabilization of the Metastable Lead Iodide Perovskite Phase via Surface Functionalization. Nano Lett. 2017, 17, 4405.

(5) Chen, T.; Foley, B. J.; Park, C.; Brown, C. M.; Harriger, L. W.; Lee, J.; Ruff, J.; Yoon, M.; Choi, J. J.; Lee, S. H. Entropy-Driven Structural Transition and Kinetic Trapping in Formamidinium Lead Iodide Perovskite. Sci. Adv. 2016, 2, e1601650.

(6) Han, Q.; Bae, S. H.; Sun, P.; Hsieh, Y. T.; Yang, Y. M.; Rim, Y. S.; Zhao, H.; Chen, Q.; Shi, W.; Li, G. et al. Single Crystal Formamidinium Lead Iodide $\left(\mathrm{FAPbI}_{3}\right)$ : Insight into the Structural, Optical, and Electrical Properties. Adv. Mater. 2016, 28, 2253.

(7) Lee, J. W.; Kim, D. H.; Kim, H. S.; Seo, S. W.; Cho, S. M.; Park, N. G. Formamidinium and Cesium Hybridization for Photo- and Moisture-Stable Perovskite Solar Cell. Adv. Energy Mater. 2015, 5, 1501310.

(8) Zhou, Y.; Kwun, J.; Garces, H. F.; Pangb, S.; Padturea, N. P. Observation of Phase-Retention Behavior of the $\mathrm{HC}\left(\mathrm{NH}_{2}\right)_{2} \mathrm{PbI}_{3}$ Black Perovskite Polymorph upon Mesoporous $\mathrm{TiO}_{2}$ Scaffolds. Chem. Commun. 2016, 52, 7273.
(9) Li, Z.; Yang, M.; Park, J. S.; Wei, S. H.; Berry, J. J.; Zhu, K. Stabilizing Perovskite Structures by Tuning Tolerance Factor: Formation of Formamidinium and Cesium Lead Iodide Solid-State Alloys. Chem. Mater. 2016, 28, 284.

(10) Zheng, X.; Wu, C.; Jha, S. K.; Li, Z.; Zhu, K.; Priya, S. Improved Phase Stability of Formamidinium Lead Triiodide Perovskite by Strain Relaxation. ACS Energy Lett. 2016, 1, 1014.

(11) Zhu, X.; Zuo, S.; Yang, Z.; Feng, J.; Wang, Z.; Zhang, X.; Priya, S.; Liu, S. F.; Yang, D. In Situ Grain Boundary Modification via Two-Dimensional Nanoplates to Remarkably Improve Stability and Efficiency of Perovskite Solar Cells. ACS Appl. Mater. Interfaces 2018, 10, 39802.

(12) Jeon, N. J.; Noh, J. H.; Yang, W. S.; Kim, Y. C.; Ryu, S.; Seo, J.; Seok, S. I. Compositional Engineering of Perovskite Materials for High-Performance Solar Cells. Nature 2015, 517, 476.

(13) Smecca, E.; Numata, Y.; Deretzis, I.; Pellegrino, G.; Boninelli, S.; Miyasaka, T.; Magna, A. L.; Albert, A. Stability of Solution-Processed $\mathrm{MAPbI}_{3}$ and $\mathrm{FAPbI}_{3}$ Layers. Phys. Chem. Chem. Phys. 2016, $18,13413$.

(14) Gu, L.; Zhang, D.; Kam, M.; Zhang, Q.; Poddar, S.; Fu, Y.; Mo, X.; Fan, Z. Significantly Improved Black Phase Stability of $\mathrm{FAPbI}_{3}$ Nanowires via Spatially Confined Vapor Phase Growth in Nanoporous Templates. Nanoscale 2018, 10, 15164.

(15) Aguiar, J. A.; Wozny, S.; Holesinger, T. G.; Aoki, T.; Patel, M. K.; Yang, M.; Berry, J. J.; Al-Jassim, M.; Zhoub, W.; Zhu, K. In Situ Investigation of the Formation and Metastability of Formamidinium Lead Tri-Iodide Perovskite Solar Cells. Energy Environ. Sci. 2016, 9, 2372.

(16) published: 17 January 2018, H.-B. F. Humidity-Induced Degradation via Grain 
Boundaries of $\mathrm{HC}\left(\mathrm{NH}_{2}\right)_{2} \mathrm{PbI}_{3}$ Planar Perovskite Solar Cells. Adv. Funct. Mater. 2018, 28, 1705363.

(17) Levchuk, I.; Osvet, A.; Tang, X.; Brandl, M.; Perea, J. D.; Hoegl, F.; Matt, G. J.; Hock, R.; Batentschuk, M.; Brabec, C. J. Brightly Luminescent and Color-Tunable Formamidinium Lead Halide Perovskite $\mathrm{FAPbX}_{3}(\mathrm{X}=\mathrm{Cl}, \mathrm{Br}, \mathrm{I})$ Colloidal Nanocrystals. Nano Lett. 2017, 17, 2765.

(18) Zhu, H.; Cai, T.; Que, M.; Song, J. P.; Rubenstein, B. M.; Wang, Z.; Chen, O. Pressure-Induced Phase Transformation and Band-Gap Engineering of Formamidinium Lead Iodide Perovskite Nanocrystals. J. Phys. Chem. Lett. 2018, 9, 4199.

(19) Weber, O. J.; Ghosh, D.; Gaines, S.; Henry, P. F.; Walker, A. B.; Islam, M. S.; Weller, M. T. Phase Behavior and Polymorphism of Formamidinium Lead Iodide. Chem. Mater. 2018, 30, 3768.

(20) Liu, Y.; Sun, J.; Yang, Z.; Yang, D.; Ren, X.; Xu, H.; Yang, Z.; Liu, S. F. 20-mm-Large Single-Crystalline Formamidinium-Perovskite Wafer for Mass Production of Integrated Photodetectors. Adv. Optical Mater. 2016, 4, 1829 .

(21) Weller, M. T.; Weber, O. J.; Frost, J. M.; Walsh, A. Cubic Perovskite Structure of Black Formamidinium Lead Iodide, $\alpha-\left[\mathrm{HC}\left(\mathrm{NH}_{2}\right)_{2}\right] \mathrm{PbI}_{3}$, at $298 \mathrm{~K}$. J. Phys. Chem. Lett. 2015, 6, 3209.

(22) Xie, L. Q.; Chen, L.; Nan, Z. A.; Lin, H. X.; Wang, T.; Zhan, D. P.; Yan, J. W.; Mao, B. W.; Tian, Z. Q. Understanding the Cubic Phase Stabilization and Crystallization Kinetics in Mixed Cations and Halides Perovskite Single Crystals. J. Am. Chem. Soc. 2017, 139, 3320 .

(23) Jiang, S.; Luan, Y.; Jang, J. I.; Baikie, T.; Huang, X.; Li, R.; Saouma, F. O.;
Wang, Z.; White, T. J.; Fang, J. Phase Transitions of Formamidinium Lead Iodide Perovskite under Pressure. J. Am. Chem. Soc. 2018, 140, 13952.

(24) Wang, P.; Guan, J.; Galeschuk, D. K.; Yao, Y.; He, C. F.; Jiang, S.; Zhang, S.; Liu, Y.; Jin, M.; Jin, C. et al. PressureInduced Polymorphic, Optical, and Electronic Transitions of Formamidinium Lead Iodide Perovskite. J. Phys. Chem. Lett. 2017, 89, 2119.

(25) Liu, G.; Kong, L.; Gong, J.; Yang, W.; Mao, H.; Hu, Q.; Liu, Z.; Schaller, R. D.; Zhang, D.; Xu, T. Pressure-Induced Bandgap Optimization in Lead-Based Perovskites with Prolonged Carrier Lifetime and Ambient Retainability. Adv. Funct. Mater. 2017, 27, 1604208.

(26) Fabini, D. H.; Stoumpos, C. C.; Laurita, G.; Kaltzoglou, A.; Kontos, A. G.; Falaras, P.; Kanatzidis, M. G.; Seshadri, R. Reentrant Structural and Optical Properties and Large Positive Thermal Expansion in Perovskite Formamidinium Lead Iodide. Angew. Chem. Int. Ed. 2016, 55, 15392 .

(27) Rehwald, W. The Study of Structural Phase Transitions by Means of Ultrasonic Experiments. Adv. Phys. 1973, 22, 721.

(28) Cordero, F.; Craciun, F.; Trequattrini, F.; Imperatori, P.; Paoletti, A. M.; Pennesi, G. Competition between Polar and Antiferrodistortive Modes and Correlated Dynamics of the Methylammonium Molecules in $\mathrm{MAPbI}_{3}$. J. Phys. Chem. Lett. 2018, 9, 4401.

(29) Sun, S.; Deng, Z.; Wu, Y.; Wei, F.; Isikgor, F. H.; Brivio, F.; Gaultois, M. W.; Ouyang, J.; Bristowe, P. D.; Cheetham, A. K. et al. Variable Temperature and High-Pressure Crystal Chemistry of Perovskite Formamidinium Lead Iodide: a Single Crystal X-Ray Diffraction and Computational Study. Chem. Commun. 2017, 53, 7537. 
(30) Xiao, G.; Cao, Y.; Qi, G.; Wang, L.; Liu, C.; Ma, Z.; Yang, X.; Sui, Y.; Zheng, W.; et al. Pressure Effects on Structure and Optical Properties in Cesium Lead Bromide Perovskite Nanocrystals. J. Am. Chem. Soc. 2017, 139, 10087.

(31) Ma, Z.; Liu, Z.; Lu, S.; Wang, L.; Feng, X.; Yang, D.; Wang, K.; Xiao, G.; Zhang, L.; Redfern, S. T. et al. PressureInduced Emission of Cesium Lead Halide Perovskite Nanocrystals. Nat. Commum. 2018, 9, 4506.

(32) Docampo, P.; Bein, T. A Long-Term View on Perovskite Optoelectronics. Acc. Chem. Res. 2016, 49, 339.

(33) Pont, S.; Bryant, D.; Lin, C. T.; Aristidou, N.; Wheeler, S.; Ma, X.; Godin, R.; Haque, S. A.; Durrant, J. R. Tuning $\mathrm{CH}_{3} \mathrm{NH}_{3} \mathrm{~Pb}\left(\mathrm{I}_{1-x} \mathrm{Br}_{x}\right)_{3}$ Perovskite Oxygen Stability in Thin Films and Solar Cells. $J$. Mater. Chem. A 2017, 5, 9553.

(34) Leguy, A. A.; Hu, Y.; CampoyQuiles, M.; Alonso, M. I.; Weber, O. J.; Azarhoosh, P.; van Schilfgaarde, M.; Weller, M. T.; Bein, T.; Nelson, J. et al. Reversible Hydration of $\mathrm{CH}_{3} \mathrm{NH}_{3} \mathrm{PbI}_{3}$ in Films, Single Crystals, and Solar Cells. Chem. Mater. 2015, 27, 3397.

(35) Gil, A.; Colchero, J.; Gómez-Herrero, J.; Baró, A. Different Stages of Water Adsorption on Au Studied by Dynamic SFM and Jumping Mode. Appl. Phys. A 2001, 72, S137.

(36) Aguiar, J. A.; Wozny, S.; Alkurd, N. R.; Yang, M.; Kovarik, L.; Holesinger, T. G.; Al-Jassim, M.; Zhu, K.; Zhou, W.; Berry, J. J. Effect of Water Vapor, Temperature, and Rapid Annealing on Formamidinium Lead Triiodide Perovskite Crystallization. ACS Energy Lett. 2016, 1,155 .

(37) Deretzis, I.; Alberti, A.; Pellegrino, G.; Smecca, E.; Giannazzo, F.; Sakai, N.;
Miyasaka, T.; Magna, A. L. Atomistic Origins of $\mathrm{CH}_{3} \mathrm{NH}_{3} \mathrm{PbI}_{3}$ Degradation to $\mathrm{PbI}_{2}$ in Vacuum. Appl. Phys. Lett. 2015, 106, 131904.

(38) Petrus, M. L.; Hu, Y.; Moia, D.; Calado, P.; Leguy, A. A.; Barnes, P. F.; Docampo, P. The Influence of Water Vapor on the Stability and Processing of Hybrid Perovskite Solar Cells Made from NonStoichiometric Precursor Mixtures. Chem. Sus. Chem. 2016, 9, 2699.

(39) Contreras-Bernal, L.; Aranda, C.; VallesPelarda, M.; Ngo, T. T.; RamosTerron, S.; Gallardo, J. J.; Navas, J.; Guerrero, A.; Mora-Sero, I.; Idígoras, J. et al. Homeopathic Perovskite Solar Cells: Effect of Humidity during Fabrication on the Performance and Stability of the Device. J. Phys. Chem. C 2018, 122, 5341. 